\title{
Genetic tests in sports medicine - many studies, little impact
}

\author{
SABINE RUDNIK-SCHÖNEBORN ${ }^{1}$
}

\begin{abstract}
Genetic research in sports has a history of more than 40 years of endeavouring to find out which genetic factors can predict the performance of an athlete. With increasing knowledge of the human genome and availability of genetic testing the market of direct-to-consumer genetic tests has exploded in recent years. There are tests for genetic variants for medical and general health issues, but there are also 'lifestyle' tests, including tests for indicators for sport performance. The human gene map for fitness and performance includes hundreds of genes that play a role in cardiorespiratory endurance, muscle strength, exercise tolerance, body composition and energy metabolism. However, there is currently no evidence that genotyping an individual is superior to traditional physiological tests in predicting athletic performance.
\end{abstract}

The impact of genetic test results is much higher in certain medical conditions which are associated with significant health risks when performing competitive sport. These are generally rare inherited disorders, mostly resulting in cardiovascular dysfunction. There are major ethical issues as to whether genetic or clinical pre-participation screenings should be applied to the population in order to prevent disease or death during sport. Some of these issues will be addressed in this article.

\section{Introduction}

Genetic research in sports medicine and the associated ethical issues have a long history. The question is whether predictive clinical or genetic screening is warranted in sports medicine. Is there a genetic profile that predicts the makings of a perfect athlete which might be used to encourage or discourage participation in specific sports? Should we try to gain a better knowledge of and access to molecular genetic testing of medical conditions which imply an increased individual health risk when performing competitive sports?

\section{Many genes with low predictability}

If you make a few clicks on the internet you can request a genetic test, using your saliva, to determine whether you are a sprinter or a marathon runner, based on a variant of the $A C T N 3$ gene.

Hard to believe? Yes, because, like many direct-to-consumer genetic tests, this test lacks sensitivity, specificity and positive predictive value. Indeed, it would be a big advantage in sports medicine if we were able to find out who has the genetic 
background to become a perfect athlete. Sports genetics research has a history of more than 40 years of trying to determine genetic factors that influence phenotypic traits as regards physical performance, training response and the metabolic adaptation of a successful athlete. Many studies have focused on the ACTN3 genotype for example, i.e. the genetic variations of the skeletal-muscle actin-binding protein $\alpha$-actinin 3 . $A C T N 3$ expression is restricted to fast muscle fibres, and the consequence of $\alpha$-actinin 3 deficiency should be a reduced sprint performance. Due to a homozygous stop codon in $A C T N 3$ (R577X) $\alpha$-actinin 3 deficiency is common in the general population, occurring with a frequency of 25 per cent in Asian and about 18 per cent in European populations. ${ }^{2}$ Following the observation in a relatively small Australian study ${ }^{3}$ that sprint athletes had a lower frequency of the $\alpha$-actinin 3 null mutation compared to controls or endurance athletes, ACTN3 testing has become commercially available.

However, subsequent studies including different disciplines and larger numbers of probands did not confirm this finding. ${ }^{4}$ Very similar expectations and observations are related to many other genes that are supposed to influence sports performance and are annually updated in the Human Fitness Gene Map. ${ }^{5}$ Hundreds of genes influence athletic performance, including those which determine skeletal muscle mass, growth and function, dynamic response to training, cardiovascular efficiency, energy metabolism, and many other factors. The conclusion after many studies is that with the current state of knowledge, the field of genetic influences on sports performance remains in its infancy. ${ }^{6}$ Since single genes have only a very limited impact on athletic performance, it is hoped to gather more information by a polygenic approach, i.e. a combined analysis of polymorphisms that play a role in either endurance, ${ }^{7}$ power $^{8}$ or metabolic pathways. ${ }^{9}$ However, there is still no evidence that any of the identified profiles have a substantial predictive value for prospectively identifying potential elite athletes. It is unlikely that genetic tests will replace traditional performance tests used to assess exercise-related variables such as oxygen uptake, running speed, or strength measurements in the near future. ${ }^{10}$ This has been recently summarised by Pitsiladis and Scott: "The current genetic evidence does not warrant genotyping an individual to establish the ability to run fast when this trait can be measured far more efficiently with a stopwatch." "So far the attempt to redesign training programmes according to the individual genetic profile - as introduced, for example, in an Australian rugby team named Sea Eagles ${ }^{12}$ on the basis of 11 exercise-related genes - seems premature and not of proven efficacy.

\section{Rare genes with high predictability}

While most phenotypic traits, either in the context of talent or in the context of disease are multifactorial, i.e. caused by many genetic and exogeneous factors, there are rare monogenic disorders where competitive sports should be avoided. These are mostly conditions affecting the heart muscle or the heart conduction system which may result in sudden cardiac death. 
Sudden cardiac deaths among young athletes have a high impact in the media and are often overestimated. The risk as a whole is low, with 2 affected athletes per 100,000 per year. ${ }^{13,14}$ A recent assessment of cardiac arrest among long-distance runners in the United States found an incidence rate of 0.54 per 100,000 participants, ${ }^{15}$ most commonly attributed to heart muscle or heart vessel disease.

Italy was the first country in the world to introduce mandatory cardiovascular preparticipation screening for athletes, including electrocardiographic examinations, in 1982. That country's experience has resulted in a common European consensus protocol for the prevention of sudden death in young competitive athletes. ${ }^{16}$ Not all European countries agree with this rigid protocol, however. ${ }^{17,18}$ It has been shown that pre-participation screening ECGs can be difficult to interpret. Mistakes in ECG interpretation could lead to high rates of inappropriate sports guidance. ${ }^{19}$ The European recommendation is that screenings should start at the beginning of competitive activity, should be repeated every two years and include medical history, physical examination, and 12-lead electrocardiogram (ECG). It is estimated that under this strategy 2 per cent of subjects affected by cardiovascular conditions would be disqualified from competitive sports and nearly 90 per cent of sudden cardiac deaths prevented $^{20}$. In the USA, meanwhile, the cost effectiveness of screening with ECG plus history and physical examination has been calculated and it has been confirmed as the preferred strategy. ${ }^{21}$ Family history is part of the screening but so far does not include genetic testing. There are some conditions for which genetic screening has been discussed:

1. Hypertrophic cardiomyopathy (HCM): This is the most frequent among the monogenic conditions in this context, affecting 1 in 500 in the population. Due to defects in different genes encoding for contractile heart proteins, affected individuals develop an increased cardiac muscle mass, often asymmetric, which reduces cardiac function and results in cardiac rhythm disturbances over time. Age at onset is generally in youth or young adulthood, i.e. an age when athletes have the most productive and competitive period. HCM has been reported to be the leading cause of sudden death in young competitive athletes, accounting for up to 40 per cent of athletic field deaths in the USA (but it occurs much less frequently in sudden deaths in Italian athletes - 2 per cent). ${ }^{22}$ The disease is autosomal dominantly inherited. This means that the presence of one mutated gene copy of the two copies available for most genes causes the disease. The mutated gene copy is passed on to the next generation with a statistical probability of 50 per cent. HCM shows a high penetrance; this means that nearly all mutation carriers develop the disease. If the defective gene copy is known to exist in a family this allows predictive genetic testing of persons at risk. Genetic screening in the general population is limited by the fact that only $60-70$ per cent of the genes causing HCM are identified, i.e. it is not possible to exclude a genetic risk in an 
individual without positive family history. This is only possible if the mutation of the affected relative is known and can be excluded in the person at risk. Therefore cascade screening of relatives in families with known mutations might be advocated. Since HCM can be detected preclinically by echocardiographic or electrocardiographic studies before cardiac function is reduced, it is a matter of debate as to whether predictive genetic testing is useful, in particular as no preventive or specific treatment is available.

2. Inherited cardiac rhythm disturbances: Abnormal cardiac conduction is associated with an increased risk for a sudden cardiac death and is caused by a variety of genetic syndromes, e.g. long QT (LQT) syndrome, short QT syndrome, Brugada syndrome, arrhythmogenic right ventricular cardiomyopathy, Wolff-ParkinsonWhite syndrome. In the Italian study this group was responsible for about 30 per cent of sudden deaths in athletes. ${ }^{23}$ Inheritance is also autosomal dominant in most families but compared to HCM, the penetrance is incomplete and prediction of cardiac complications far from precise. Molecular genetic screening in athletes without a medical or family history will also be hampered by a large number of genes causing the same disease, so called genetic heterogeneity, and a large proportion of unclassified genetic variants which might be of clinical significance but can also represent harmless polymorphisms. Following the protocol of the European Society of Cardiology ${ }^{24}$ the consequence of genetic screening would be to exclude possible gene carriers from participating in competitive sport without being able to specify the overall risk. A negative result of genetic screening, on the other hand, does not exclude risk for an inherited heart disease, taking into account that many genes remain to be identified and that not all mutations can be covered in a testing panel.

Medical intervention in genetic arrhythmia syndromes generally starts when the defined ECG abnormalities are detected, ${ }^{25}$ a time point which cannot be predicted by mutation analysis. The risk of serious cardiac events ranges from 50 per cent up to the age of 15 years in some mutation types of the $K C N Q 1$ gene causing LQT1 ${ }^{26}$ to less than a 10 per cent lifetime risk in Brugada syndrome. ${ }^{27}$ A situation in clinical practice might be envisaged as follows: A young athlete has a family history of a cardiac arrest in a cousin who has a mutation in the $S C N 5 A$ gene, which can explain about 20 per cent of patients with Brugada syndrome. Given that this mutation has been transmitted in the family through several generations without causing trouble (except for the cousin) and can also be detected in our athlete, should he be banned from competitive sports or not?

Moreover, cardiac events in LQT syndromes are associated with gene-specific triggers, in that events in LQT1 are triggered by exercise and so are particularly dangerous for athletes, while most events in LQT2 and LQT3 occur during sleep or rest. Therefore risk stratification in cardiac rhythm disturbances has to take the 
specific type of arrhythmia, the cumulative probabilities for adverse events, the mutation type and location and the physical activity of an athlete into account.

\section{Few genes with moderate predictability}

Athletes in high-contact sports, such as boxing, ice-hockey or rugby, have a higher risk of brain damage. Following the observation that chronic traumatic brain injury in boxing was associated with a 50 per cent increase in rates of Alzheimer's disease in a small sample of boxers, ${ }^{28}$ a debate began as to whether athletes should undergo genetic testing for factors that predispose to Alzheimer's disease. Rates of Alzheimer's disease increase dramatically with age. While only 1-2 per cent of individuals develop the disease between 65 and 70 years, this proportion increases to 10-20 per cent in the age group between 85 and 90 years. ${ }^{29}$ The underlying causes of the disease are still not fully understood, but it is known that the genetic information given by the apolipoprotein $\mathrm{E}(A P O E)$ gene can influence the risk. The $A P O E$ gene is present in three variants in the population, $\varepsilon 2, \varepsilon 3$, or $\varepsilon 4$. In the presence of one $\varepsilon 4$ copy, the age-related risk of developing Alzheimer's disease is 3-fold; if both gene copies belong to the $\varepsilon 4$ variant, this risk is increased to $10-15$-fold. ${ }^{30}$

While one study of college athletes did not show an important association between carrying the $A P O E \& 4$ variant and sustaining a concussion, ${ }^{31}$ outcome after head injury seems to be influenced by $A P O E .^{32}$ If an athlete with at least one $A P O E \varepsilon 4$ gene copy starts a competitive career as a boxer, his individual risk for Alzheimer's disease or a poor outcome after head injury is most likely increased and it might be important to advise him in this respect. However, since there is no prevention or treatment of Alzheimer's disease at present, the information about an increased risk for late onset dementia in a young athlete might be potentially harmful for his quality of life, in terms of medico-legal, employment and insurance issues. As such no guidelines exist on how to deal with a possible increased risk for Alzheimer's disease in sports at risk for brain damage, nicely summarised by Alla Katsnelson: "Gene tests for brain injury (are) still far from the football field." ${ }^{33}$

\section{Conclusion}

On the basis of our current knowledge, genetic screening to predict athletic performance is not recommended. As long as clinical assessments or traditional performance tests are superior, genetic testing should only be permitted at the request of the individual who will be tested. Following the guidelines of the European Society of Human Genetics (ESHG) ${ }^{34}$ commercially available tests for genetic variants in the context of general health purposes, life-style and sport performance should only be offered if the clinical utility and validity of the analysis is proven, if informed consent is given, and if privacy and confidentiality are secured. Since genetic results can reduce quality of life and can have significant socioeconomic consequences, these tests should be confined to mature individuals and the right not-to-know should be respected. Special measures should be taken to avoid inappropriate testing of minors. 
In families at risk for rare monogenic disorders associated with an increased death rate in competitive sports, individual decision-making about predictive genetic testing is warranted. The German Genetic Diagnosis Act which was introduced in $2010^{35}$ has strict regulations as regards genetic testing, specifically in minors. In the case of predictive genetic testing, genetic counselling is mandatory under the Act. Predictive genetic testing is allowed in minors only if there are options for prevention or treatment or if potential harmful investigations can be avoided. For diseases that start in adulthood, genetic tests should be confined to mature individuals who fully understand the relevant issues. Similar recommendations were made by the ESHG. ${ }^{36}$ Internationally and nationally approved guidelines are required for the implementation of new biomedical research results into clinical practice in an ethically acceptable manner.

\footnotetext{
${ }^{1}$ Institute for Human Genetics, Medical Faculty, RWTH Aachen University, Germany. srudnikschoeneborn@ukaachen.de

${ }^{2} \mathrm{~N}$. Yang et al. ACTN3 genotype is associated with human elite athletic performance. Am J Hum Genet 2003; 73: 627-631.

3 Ibid.

${ }^{4} \mathrm{~T}$. Alfred et al. ACTN3 genotype, athletic status, and life course physical capability: meta-analysis of the published literature and findings from nine studies. Hum Mutat 2011; 32: 1008-1018.

${ }^{5} \mathrm{M}$. S. Bray et al. The Human Gene map for performance and health-related fitness phenotypes: the 2006-2007 update. Med \& Sci Sports Exerc 2009; 41: 35-73.

${ }^{6}$ Z. Puthucheary et al. Genetic influences in sport and physical performance. Sports Med 2011; 41: 845-859.

${ }^{7}$ A. G. Williams \& J. P. Folland. Similarity of polygenic profiles limits the potential for elite physical performance. $J$ Physiol 2008; 586: 113-121.

${ }^{8}$ J. R. Ruiz et al. Can we identify a power-orientated polygenic profile? J Appl Physiol 2010; 108: 561566.

${ }^{9}$ I. I. Ahmetov et al. The combined impact of metabolic gene polymorphisms on elite endurance athlete status and related phenotypes. Hum Genet 2009; 126: 751-761.

${ }^{10}$ A. G. Williams et al. Genetic research and testing in sport and exercise science. British association of sport and exercise sciences position stand, 2007. www.bases.org.uk/BASES-Expert-Statements.

${ }^{11}$ Y. P. Pitsiladis, R. Scott. Essay: the makings of a perfect athlete. The Lancet 2005; 366: S16-17.

${ }^{12}$ C. Dennis. Rugby team converts to give gene tests a try. Nature 2005; 434:260.

${ }^{13}$ B. J. Maron. Hypertrophic cardiomyopathy and other causes of sudden cardiac death in young competitive athletes, with considerations of preparticipation screening and criteria for disqualification. Cardiol Clin 2007; 25: 399-414.

${ }^{14}$ J. A. Drezner et al. Survival trends in the United States following exercise-related sudden cardiac arrest in the youth: 2000-2006. Heart Rhythm 2008; 5: 794-799.

${ }^{15}$ J. H. Kim et al. Cardiac arrest during long-distance running races. NEJM 2012; 366: 130-140.

${ }^{16} \mathrm{D}$. Corrado et al. Cardiovascular pre-participation screening of young competitive athletes for prevention of sudden death: proposal for a common European protocol. Eur Heart J 2005; 26; 516-524.

${ }^{17}$ A. Mosterd, B. K. Velthuis. Screening of athletes is undesirable. Ned Tijdschr Geneeskd 2012; 156 : A5001.

${ }^{18}$ C. Wren. Comment on Cardiovascular pre-participation screening of young competitive athletes for prevention of sudden cardiac death: proposal for a common European protocol. Eur Heart J 2005; 1804-1811.
} 
${ }^{19}$ A. C. Hill et al. Accuracy of interpretation of pre-participation electrocardiograms. J Pediatr 2011; 159: 783-788.

${ }^{20} \mathrm{D}$. Corrado et al. Trends in sudden cardiovascular death in young competitive athletes after implementation of a preparticipation screening program. JAMA 2006; 296: 1593-1601.

${ }^{21} \mathrm{M}$. T. Wheeler et al. Cost effectiveness of pre-participation screening for prevention of sudden cardiac death in young athletes. Ann Inter Med 2010; 152: 276-286.

${ }^{22}$ D. Corrado et al., op. cit. note 13

${ }^{23}$ Ibid.

${ }^{2424}$ A. Pelliccia et al. Recommendations for competitive sports participation in athletes with cardiovascular disease. Eur Heart J 2005; 26: 1422-1445.

${ }^{25} \mathrm{ACC} / \mathrm{AHA} / \mathrm{ESC} 2006$ guidelines for management of patients with ventricular arrhythmias and the prevention of sudden cardiac death. JACC 2006; 48: e247-e346

${ }^{26}$ A. Barshehet et al. Genetics of sudden cardiac death. Curr Cardiol Rep 2011; 13: 364-376.

${ }^{27}$ S. G. Priori. Risk stratification in Brugada syndrome. J Am Coll Cardiol 2012; 59: 37-45.

${ }^{28}$ B. D. Jordan et al. Apolipoprotein E epsilon4 associated with chronic traumatic brain injury in boxing. J Am Med Assoc 1997; 278; 136-140.

${ }^{29}$ S. Seshadri, P. A. Wolf. Lifetime risk of stroke and dementia: current concepts, and estimates from the Framingham study. Lancet Neurol 2007; 6: 1106-1114.

${ }^{30}$ E. Genin et al. APOE and Alzheimer's disease: a major gene with semi-dominant inheritance. $\mathrm{Mol}$ Psychiatr 2011; 16: 903-907.

${ }^{31}$ V. L. Kristman et al. Does the apolipoprotein epsilon 4 allele predispose varsity athletes to concussion? Clin J Sport Med 2008; 18: 322-328.

${ }^{32} \mathrm{G}$. M. Teasdale et al. The association between APOE $\varepsilon 4$, age and outcome after head injury: a prospective study. Brain 2005; 128: 2556-2561.

${ }^{33}$ A. Katsnelson. Gene tests for brain injury still far from the football field. Nat Med 2011; 6: 638 .

${ }^{34}$ European Society of Human Genetics: Statement of the ESHG on direct-to-consumer genetic testing for health related purposes. Eur J Hum Genet 2010; 18: 1271-1273.

${ }^{35} \mathrm{http}: / / \mathrm{www}$.gesetze-im-internet.de/gendg/index.html (accessed 8 September 2012).

${ }^{36}$ European Society of Human Genetics: Genetic testing in asymptomatic minors: recommendations of the European Society of Human Genetics. Eur J Hum Genet 2009; 17: 720-721. 